\title{
The acoustic properties of snores
}

\author{
R. Beck*, M. Odeh**, A. Oliven**, N. Gavriely ${ }^{+}$
}

The acoustic properties of snores. R. Beck, M. Odeh, A. Oliven, N. Gavriely. OERS Journals Ltd 1995.

ABSTRACT: This study was undertaken in an attempt to characterize the acoustic properties of snoring sounds in the time and frequency domains, and to correlate between these properties and the mechanical events underlying their production.

Three experimental set-ups were used: 1) Dog model - six mongrel dogs, in which partial upper airway obstruction was created by an implanted supraglottic balloon. Flow, supraglottic pressure, and snoring sounds were recorded during different degrees of obstruction. Fifteen to 20 snores from each dog (total 100 snores) were analysed. 2) Simulated human snores - Six simulated snores from each of four subjects were recorded in two locations (trachea and ambient) with simultaneous airflow, and their correlations examined. 3) Snoring patients - snores were recorded with an ambient microphone from nine subjects with "heavy" snoring and no obstructive sleep apnoea (OSA). Forty to 50 snores from each subject were analysed (total of 400 snores). The snoring sound was analysed in the time (time-expanded waveform) and frequency (power spectrum) domains.

After analysing these snores, we were able to identify two dominant patterns which are distinctly different from each other: the "simple-waveform" and the "complexwaveform". The complex-waveform snore is characterized by repetitive, equallyspaced, train of sound structures, starting with a large deflection followed by a decaying amplitude wave. In the frequency domain, it is characterized by multiple, equally-spaced peaks of power (comb-like spectrum). Simple-waveform snores have a quasi-sinusoidal waveform, with a range of variants, and almost no secondary internal oscillations. Their power spectrum contains only 1-3 peaks, of which the first is the most prominent. We developed a mathematical representation of these waveforms, which is presented along with its implications.

The complex-waveform snores result from colliding of the airway walls and represent actual brief airway closure. Simple-waveform snores are of higher frequency and probably result from oscillation around a neutral position without actual closure of the lumen.

Eur Respir J., 1995, 8, 2120-2128.
*Respiratory Division, Dept of Pediatrics, University of Calgary, and the Alberta Childrens Hospital, Calgary, Alberta, Canada. **Dept of Medicine "B", Bnei Zion Hospital, Haifa, Israel. ${ }^{+}$Pulmonary Physiology Unit, Faculty of Medicine, Technion-Israel Institute of Technology, and the Rappaport Family Institute for Research in the Medical Sciences, Haifa, Israel.

Correspondence: N. Gavriely

Pulmonary Physiology Unit

Faculty of Medicine

Technion-Israel Institute of Technology

Efron Street

P.O. Box 9697

Haifa

Israel 31096

Keywords: Obstructive sleep apnoea

snoring

sound analysis

upper airways

Received: January 121995

Accepted after revision August 141995
Snoring is a most important symptom connected with the obstructive sleep apnoea (OSA) syndrome, which is associated with significant morbidity, such as hypertension, cor pulmonale, behavioural and personality changes and daytime somnolence. Snoring is also a frequent reason for seeking medical attention, being the cause of much disruption to other family members, as well as to the snorer. Snoring is a very common symptom. It is more common in males than in females, and in overweight people of both sexes. Its prevalence rises markedly after the age of $40 \mathrm{yrs}$, with $63 \%$ of males and $44 \%$ of females being habitual snorers [1]. Current data suggest that snoring might represent the first stage of the OSA syndrome [2].

Investigation of snoring and possible OSA presently involves a sophisticated procedure, polysomnography, whereby multiple physiological parameters are monitored during a night's sleep in a specially equipped laboratory. Presence and sound level of snoring is usually recorded, but the snoring sound is not analysed acoustically, as no practical implications to such analysis have yet been found.

Acoustic analysis of snores can theoretically be used to obtain much useful information, in a similar manner to acoustic analysis of speech. Characterizing the snoring sound in the time and frequency domains, may allow recognition of different patterns which depend on the mode or place of sound production. Constructing transfer functions [3] or calculations derived from an upper airway model [4] might allow identification of the site of the snore production and with it the site of obstruction. Modern signal analysis equipment and software now enable very rapid sound recording and analysis. With the use of high speed digitizers, powerful computers and appropriate software, sound can be recorded directly into the computer and analysis functions performed on-line, often breath-by-breath. This opens possibilities, such as automatic detection of specific acoustic events.

Snoring is thought to result from vibration of the soft palate and adjacent tissues [5, 6]. However, it is 
unclear how these vibrations produce the snoring sound. Studies have shown pressure and flow oscillation during simulated snoring $[6,7]$. The frequency as well as the waveforms of these oscillations were similar to sound frequencies and waveforms that we have observed in snores (unpublished data). This led us to postulate that it may be possible to correlate between the sound characteristics of the snore and the mechanical events underlying it. Further possible applications of sound analysis of snores include identification of certain characteristics enabling inclusion or exclusion of certain diagnostic categories from the differential diagnosis, and the possibility of follow-up after treatment. Recording of snores is simple and noninvasive, requiring only a microphone hung above the patient's head, and can be performed in the patient's home.

We undertook the present study in order to clarify the acoustic properties of snores in the time and frequency domains. We found that snoring sounds are characterized by repetitive sequences of sound structures, occurring at $62-136 \mathrm{~Hz}$, and which contain large low-frequency waveforms interwoven with rapid oscillation. When transformed into the frequency domain, these sound structures appear as multiple, closely-spaced peaks of power centred around the internal frequency of the individual sound structures. These data were compared to the outcome of a simple mathematical model of the snoring waveform.

\section{Methods}

Three experimental set-ups were used in this study: a dog model of upper airway obstruction; simulated snores in normal volunteers; and snores recorded during sleep in a sleep laboratory from subjects with heavy snoring but no OSA.

\section{Dog model}

Figure 1 illustrates the experimental set-up of the dog model. Six mongrel dogs, weighing $17.2 \pm 3.5 \mathrm{~kg}$ (mean $\pm \mathrm{SD}$ ), were studied whilst undergoing an experiment to determine the function of the upper airway dilating muscles. The dogs were anaesthetized with pentobarbital, $30 \mathrm{mg} \cdot \mathrm{kg}^{-1}$. Further doses were given as required to maintain a level of deep anaesthesia without suppressing spontaneous breathing. Venous and arterial lines were established in femoral or forelimb vessels. A low tracheostomy was performed, in such a manner as to divert all tracheal flow through a Fleisch No. 2 pneumotachograph which was placed between the proximal and the distal limbs of the tracheostomy. The pneumotachograph was calibrated against a Morgan Spiroflow spirometer. A thin pressure catheter (polyethylene, $2 \mathrm{~mm}$ internal diameter) was inserted through the upper limb of the tracheostomy and the tip placed in the hypopharynx to measure upper airway pressure $(P$ uaw $)$. The pressure catheter was connected to a differential pressure transducer (Validyne MP-45, $\pm 50 \mathrm{cmH}_{2} \mathrm{O}$ ). Upper airway obstruction was created with a small rubber balloon,

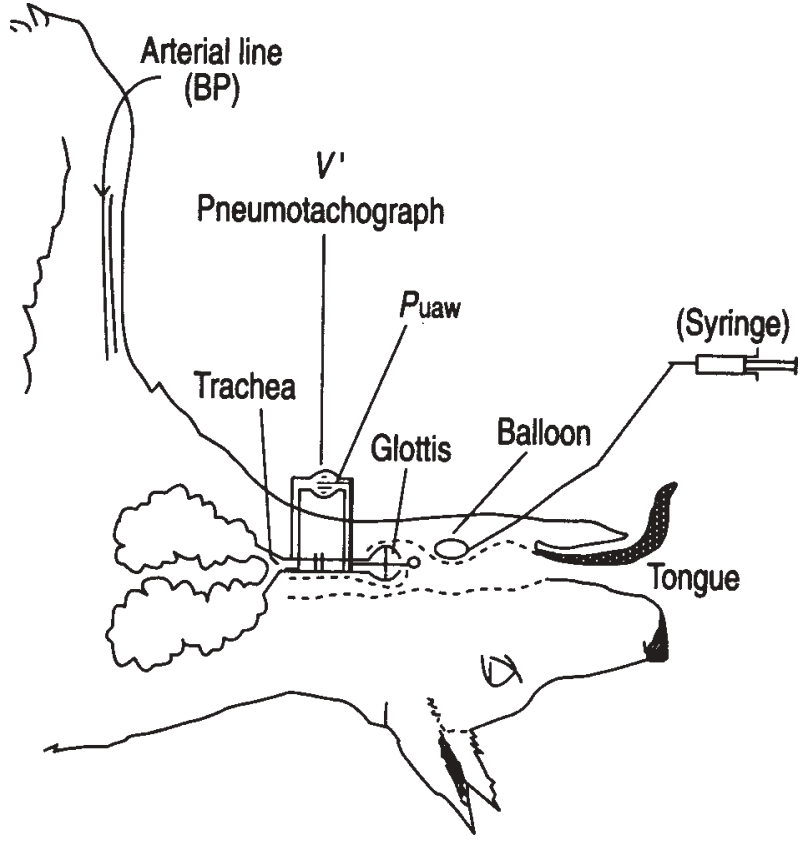

Fig. 1. - Schematic graphical representation of the dog model of upper airway obstruction. Upper airway obstruction is created by stepwise inflation of the balloon. Upper airway pressure $(P$ uaw $)$ is measured on the alveolar side of the obstruction site. Airflow $\left(V^{\prime}\right)$ is measured by a pneumotachograph inserted between two limbs of a tracheostomy. The dog is breathing spontaneously. BP: blood pressure.

which was inserted through a skin incision in the neck and tunnelled to a position about $1 \mathrm{~cm}$ above the larynx. The wound was then sutured and varying degrees of obstruction were obtained by inflating the balloon with different quantities of air. The amount of air needed to obtain three levels of obstruction (mild, moderate, severe) was determined for each dog by the clinical response (respiratory effort, tachypnoea), and the same amounts were always used in repeated experiments on the same dog. A fourth level of obstruction (complete) was also created, but data are not included, as no sounds were produced.

The $P$ uaw and flow $\left(V^{\prime}\right)$ signals were recorded on a chart recorder (Graphtec Linearecorder Mark VII WR3101, Japan). Snoring sounds were recorded with a HewlettPackard HP21050A contact sensor placed on the lateral aspect of the neck above the tracheostomy and held in place with an elastic band. Sounds were amplified $(\times 10-100)$, band filtered $\left(75-2,000 \mathrm{~Hz}, 20 \mathrm{~dB} \cdot\right.$ oct $\left.^{-1}\right)$ and digitized (8-bit A/D converter, SoundWave ${ }^{(\mathbb{m}}$, Minneapolis, MA, USA) at $5.5 \mathrm{kHz}$ into a Macintosh SE computer (Apple Computer Inc., Cupertino, CA, USA) using the SoundWave ${ }^{\mathbb{I}}$ software. The snoring sound analysis was performed in the time domain by time-expanded waveform analysis, and in the frequency domain by sound spectrum using a 512-point fast fourier transform (FFT). This sound analysis was performed off-line, on 15-20 snores from each dog. The study was approved by the Technion Helsinki committee for animal experiments.

\section{Simulated snores}

Four healthy male nonsnoring subjects (aged 29-43, mean $35 \pm 7$ (SD) yrs) were asked to simulate snoring in the 
laboratory. The subjects were in the sitting position with noseclips on and inhaling through a calibrated Fleisch No. 2 pneumotachograph with a round mouthpiece, connected to a differential pressure transducer (Validyne MP-45 $\pm 50 \mathrm{cmH}_{2} \mathrm{O}$ ). Tight lip seal around the mouthpiece was ensured, so that the subjects were inhaling (and snoring) through their mouth only. Snoring was recorded in inspiration only, in two locations: 1) Ambient - an electret-type condenser microphone (model EM-110, Altai, Japan) hung in front of the pneumotachograph, $20 \mathrm{~cm}$ from the mouth; 2) Tracheal - a piezoelectric contact sensor (Rappaport Institute, Haifa, Israel, linear response $70-2,000 \mathrm{~Hz}$ ) placed over the trachea $2 \mathrm{~cm}$ above and slightly to the right of the suprasternal notch. These sounds were amplified $(\times 200)$ and band filtered (75-2,000 Hz, Custom Design PPG Lung Sounds Amplifier/Filter, Rappaport Institute, Haifa, Israel). The flow and snoring sounds signals were digitized with a 12-bit, 8-channel A/D converter (MacADIOS 8ain Data Acquisition System, GW Instruments, Somerville, MA, USA) and appropriate software (SuperScope ${ }^{\mathbb{}}{ }^{-}$Data Acquisition Software, GW Instruments, Somerville, MA, USA), at $5,760 \mathrm{~Hz}$, into a Macintosh SE computer. The data were analysed off-line. Sound analysis in the time domain (time-expanded waveform) and frequency domain (sound spectrum) by 2,048-point FFT was performed off-line. The study was approved by the Technion Ethics Committee, and informed consent was obtained from all subjects.

\section{"Natural" snores}

Snoring was recorded during sleep from subjects undergoing polysomnography at the Technion Sleep Laboratory. The subjects were eight males and one female, aged 30-70 yrs (mean 51 \pm 13 (SD) yrs). All were referred for investigation because of heavy/loud snoring but none had a history of apnoea being observed or documented. Eight of the patients underwent standard polysomnography, which included inductive plethysmography, electrocardiography (ECG), electroencephalography (EEG), electrooculography (EOG), electromyography (EMG), nasal thermister, and sound amplitude detector. None had invasive monitoring and no continuous positive airway pressure (CPAP) was applied. One of the subjects (female, aged 70 yrs) was recorded at her home. Snoring was recorded with an ambient electret-type condenser microphone (model EM-110, Altai, Japan, or model 33-1052, Radio Shack, Tandy Corp., Fort Worth, TX, USA). The microphone was hung $40-50 \mathrm{~cm}$ above the head of the sleeping subject. The sounds were amplified (×5,000-10,000) and band filtered $(75-2,000 \mathrm{~Hz}$, Custom Design PPG Lung Sounds Amplifier/Filter, Rappaport Institute, Haifa, Israel) and recorded on Hi-Fi tape (AIWA stereo cassette deck AD-F360). The sounds were analysed off-line by digitizing at $9,350 \mathrm{~Hz}$ into a Macintosh SE computer using the MacADIOS 8ain A/D converter and SuperScope software, as described above. Analysis was performed in the time domain (time-expanded waveform) and frequency domain (sound spectrum by 2,048-point
FFT. The study was approved by the Technion Ethics Committee, and informed consent was obtained from all subjects.

\section{Signal processing}

The MacADIOS 8ain Data Acquisition hardware (GW Instruments, Somerville, MA, USA) is an 8-channel analog input, 12-bit, $14 \mu \mathrm{s} \mathrm{A/D} \mathrm{converter.} \mathrm{Sampling} \mathrm{rate}$ can be adjusted to need, with maximal overall sampling capacity of 28,800 samples $\cdot \mathrm{s}^{-1}$. SuperScope Data Acquisition Software (GW Instruments, Somerville, MA, USA) enables simultaneous display of up to 50 waves, which can be either of actual data input or mathematical functions of these data. With the use of a fast computer, functions can be executed "on-line" (for example, "breathby-breath" power spectra of breathing sounds). Figure 2 is a schematic illustration of the snoring sound recording and analysis system. The details vary and are described with each experiment.

\section{Results}

\section{Dog model}

All six dogs produced snoring sounds when partial upper airway obstruction was created by inflating the balloon. Flow and $P$ uaw tracings during partial upper airway obstruction showed, in all runs, a plateau in inspiratory flow, accompanied by marked pressure and flow oscillation. Snoring sounds were always associated with these flow and $P$ uaw oscillations.

\section{Snoring sound analysis}

Figure 3 shows representative examples of snoring sounds from the dog model, in the time and frequency

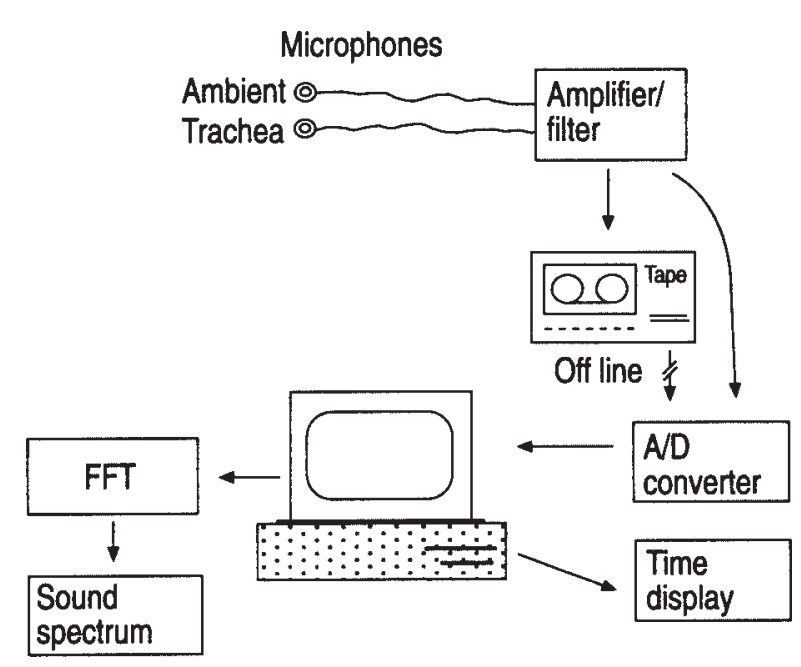

Fig. 2. - Schematic illustration of the sound recording system. Sounds can be recorded into a tape for future off-line analysis, or directly digitized into the computer. Analysis is performed in the time domain and frequency domain (power spectrum). 
a)
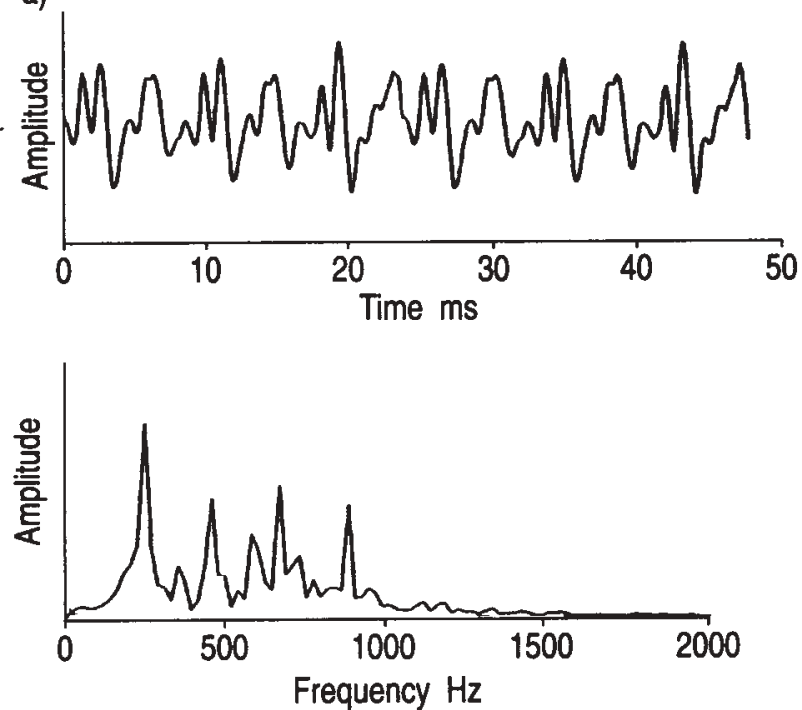

b)
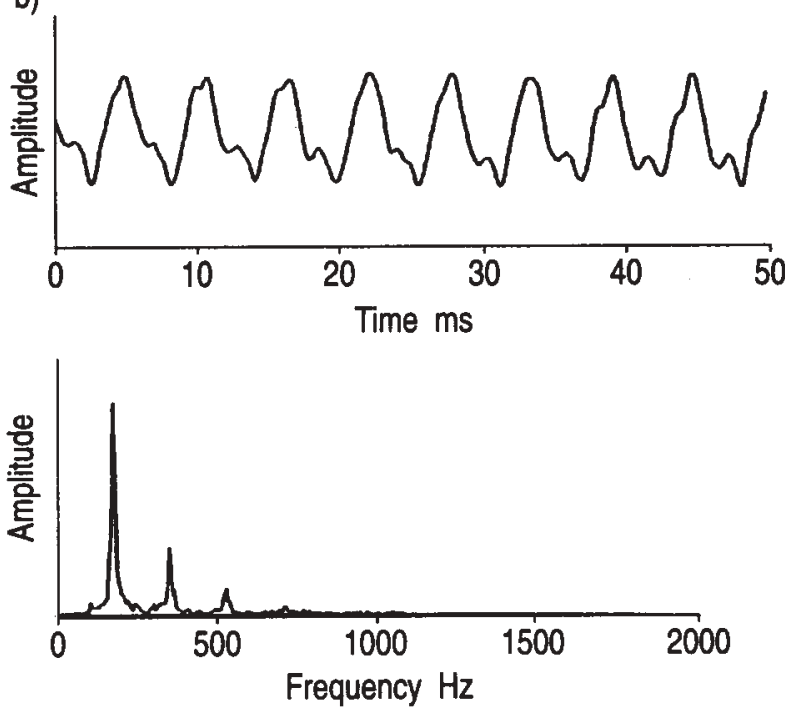

Fig. 3. - Examples of snoring sounds from the dog model. a) "Complex-waveform". The upper panel shows the time domain. Note the "complex" but repetitive appearance of the waveforms, which are each made up of 5-6 faster waves or deflections. The lower panel shows the frequency domain (power spectrum), with a number of power peaks of various amplitudes. b) "Simple-waveform". The upper panel shows the time domain. The sound wave is an asymmetric periodic oscillation. The lower panel shows the power spectrum - a large peak, with smaller harmonics at higher frequencies.

domains. Fifteen to 20 snores were analysed from each dog (a total of 100 snores). After analysing all the snores, we could identify two distinct sound patterns: 1) Segments of the snoring sound which were characterized in the time domain by repetitive complex sound structures (fig. 3a, upper panel). The frequency of these structures was low, 64-135 Hz. The structures were composed of a number (3-8) of waves of higher frequency $(220-850 \mathrm{~Hz})$, which occurred with a constant shape, amplitude and frequency to form these uniformly shaped slow "sound structures". In the frequency domain, these snoring sounds appeared as multiple, closely-spaced peaks of power, resembling a comb-like structure (fig. 3a, lower panel). The position of the highest peak represented the frequency of the fast oscillations within a sound structure, whereas the frequency interval between each of the multiple comb-like peaks represented the number of sound structures per second (mean frequency $88 \pm 17$ (SD), range $64-135 \mathrm{~Hz}$ ). These sound structures were termed" complex-waveform snores". 2) Segments of sound which appeared more sinusoidal in the time domain and which, on frequency analysis, showed a prominent peak of power with a small number of harmonics (fig. 3b). These signals had higher frequencies (mean $232 \pm 25$ (SD), range 174-292 Hz). These waveforms were termed "simplewaveform snores". More than $60 \%$ of snores fell into one of these two categories. The majority of the other sound segments showed, in the frequency domain, multiple closely spaced peaks of power, but in the time domain the waves were more irregular and hard to characterize systematically.

\section{Simulated snores}

Figure 4 shows a representative simultaneous tracing of flow $\left(V^{\prime}\right)$ and sound waves recorded from the tracheal sensor $(\mathrm{S})$ and the ambient microphone $(\mathrm{M})$ from each of the four subjects. Six runs were performed in each subject, and all results were similar to the ones shown, demonstrating oscillation of identical frequency in flow and both sound waveforms. All snoring sounds in this experiments showed, in the time domain, repetitive complex sound structures, i.e. complex-waveform snores. The shape of the waves differed between different runs, but the above finding always remained. The highest and sharpest sound wave deflection always occurred when oscillation in inspiratory flow signal was at its highest, i.e. the most negative (fig. 4, panels 1a-4a). The snoring sound waves from the tracheal sensor and ambient microphone, though of different shape, both always showed repetitive complex sound structures, similar to the ones seen in the dog model. The frequency of the sound structures was identical in the two sites, and there was no phase shift, i.e. the highest and sharpest sound wave deflection occurred at the same time. The initial highest sound wave deflection, however, was always in opposite directions in these two recordings, i.e. negative in the microphone (mouth) and positive in the tracheal sensor (fig. 4, panels 1a-4a). This polarity was constant in all sound recordings, reflecting the fact that the sound source was situated between the two microphones. In the frequency domain, all these sounds translated into multiple, closelyspaced (comb-like) peaks of power, ranging $50-800 \mathrm{~Hz}$ (fig. 4, panels $1 b-4 b$ ).

\section{"Natural" snores}

From each subject, 40-50 snores were analysed (a total of 400 snores). Two distinct patterns of sound were recognized, and were similar to those described for the dog model and the simulated snores: 1) Sounds characterized in the time domain by repetitive complex sound structures, which occur at a low frequency (mean $98 \pm 16$, 
1a)

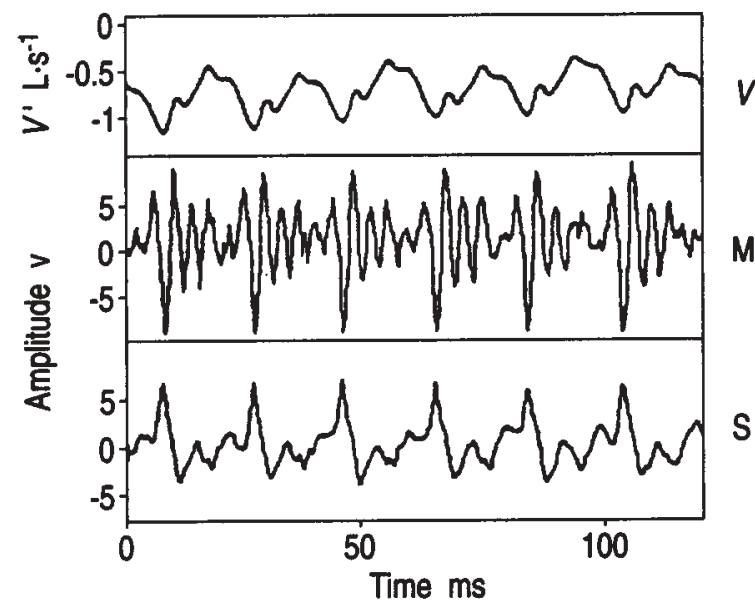

1b)

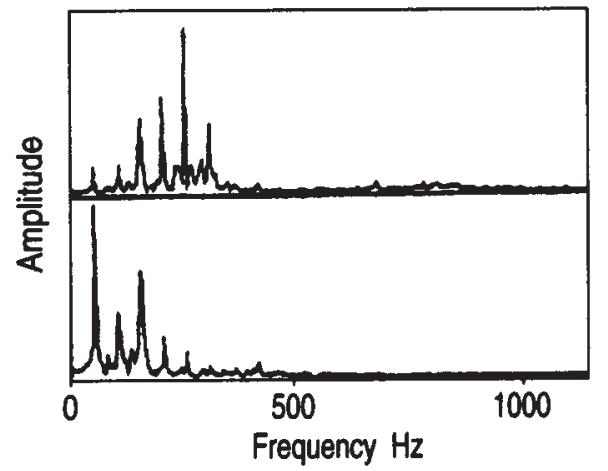

3a)

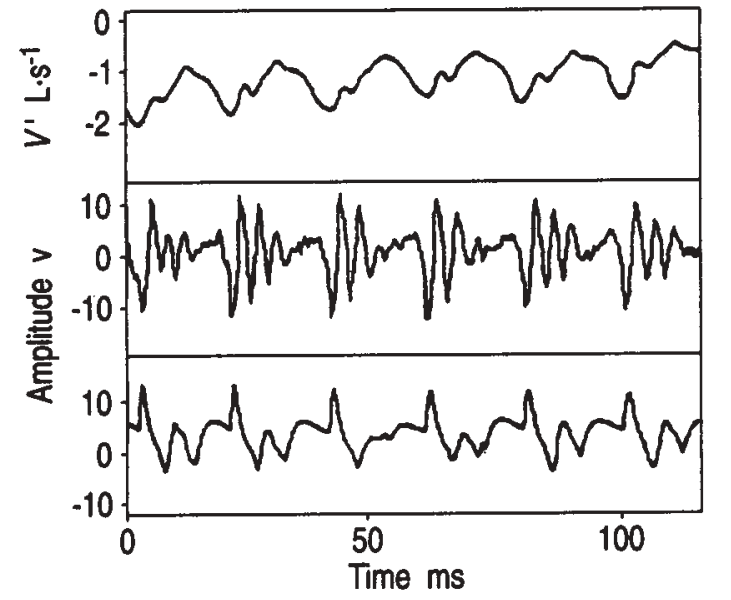

3b)

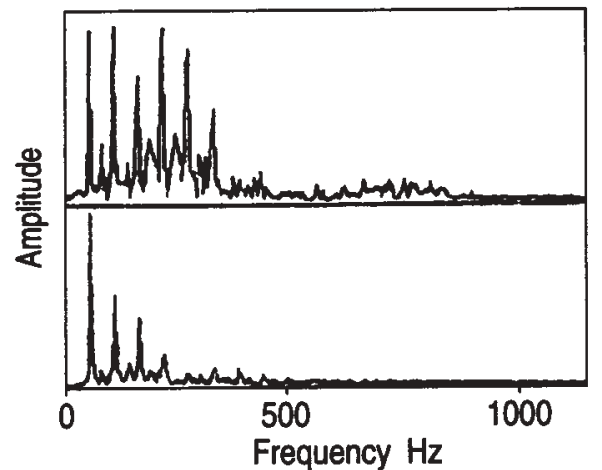

2a)

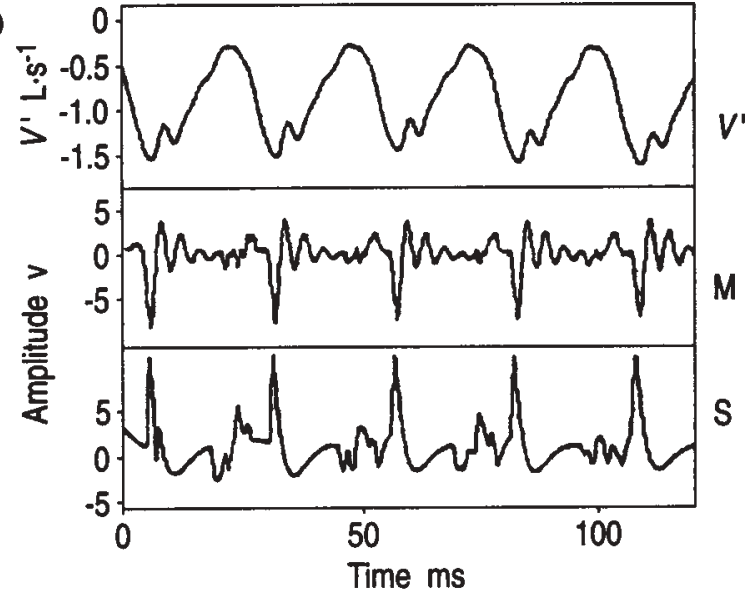

2b)

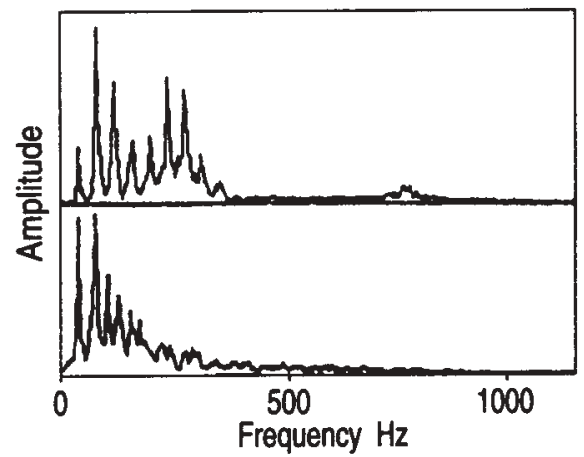

M

4a)

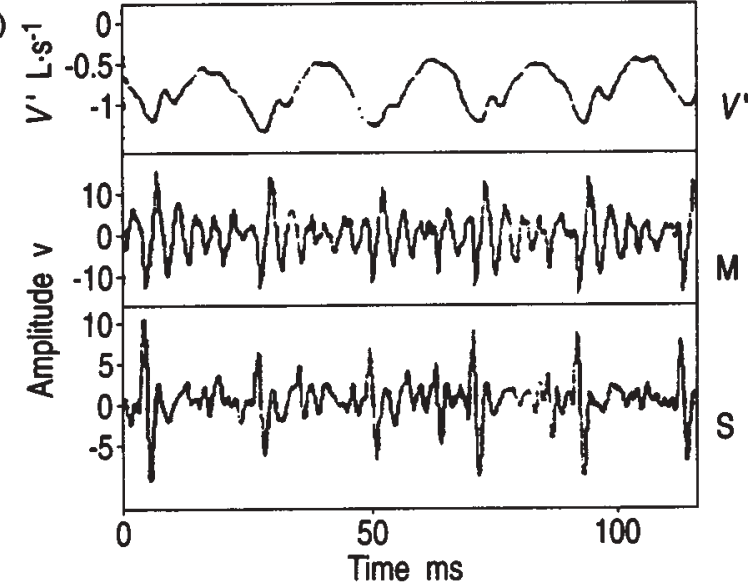

4b)

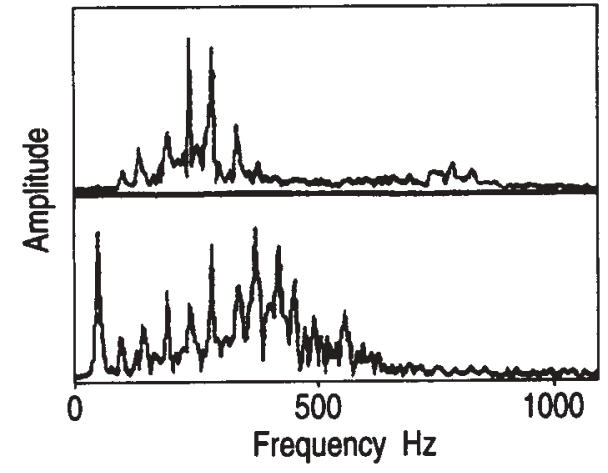

M

S

Fig. 4. - Panels 1-4 show representative simultaneous tracings of a) flow $\left(V^{\prime}\right)$ and sound waves from ambient microphone (M) and tracheal sensor (S) plotted against time and b) amplitude plotted against frequency from each of the four subjects. Note the highest sound wave deflection coinciding with the highest flow signal. 
a)
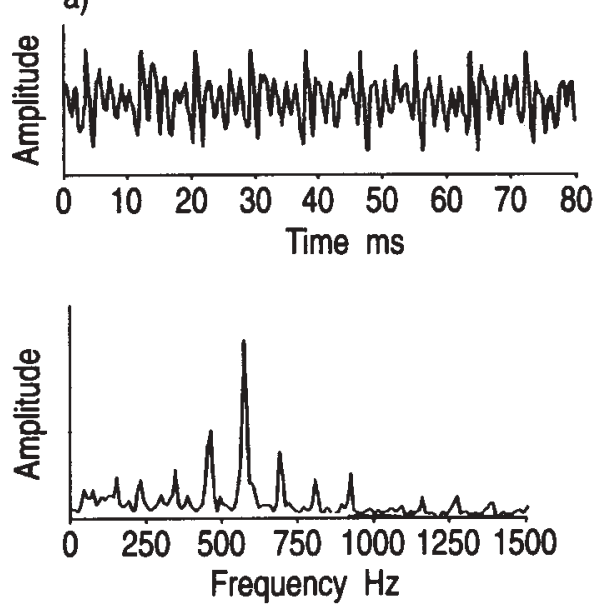

b)
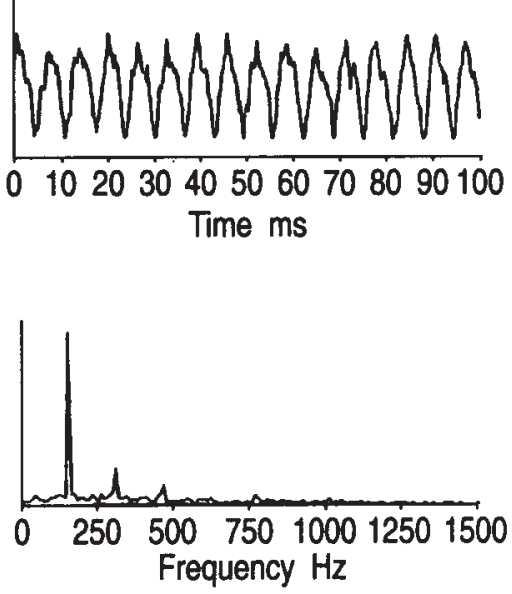

c)
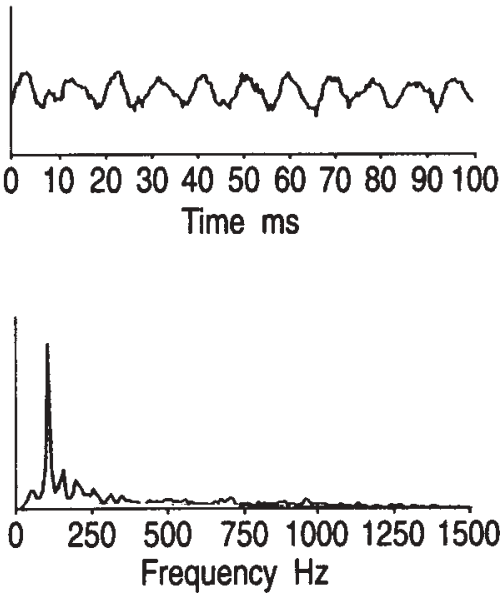

Fig. 5. - Examples of typical snoring sounds from subjects with heavy snoring but no obstructive sleep apnoea (OSA), recorded with an ambient microphone. The upper panels show the time domain and the lower panels the frequency domain. a) "Complex-waveform" showing a repetitive sound structure comprised of five internal waves (upper panel) and multiple peaks of power in its sound spectrum (lower panel). b) Asymmetric "simple-waveform", with a power spectrum of a dominant spike and a few harmonics. c) Sinusoidal "simple-waveform", with a single power peak on spectral analysis. The fine oscillation superimposed on the wave represents some "white" environmental noise.

range $62-136 \mathrm{~Hz}$ ) and are composed of $3-7$ waves of higher frequency (mean 602 \pm 172 , range $240-970 \mathrm{~Hz}$ ), i.e. complex-waveform snores. In the frequency domain these sounds always appeared as multiple peaks of different amplitudes (fig. 5a). 2) Sound waves of "simpler" appearance, either sinusoidal or an asymmetric slow waveform, with a frequency range of $105-238 \mathrm{~Hz}$ (mean $168 \pm 27 \mathrm{~Hz}$ ), i.e. simple-waveform snores. In the frequency domain these appear as a single large peak of power, often with a small number of lower amplitude harmonics (fig. 5b and 5c). One of the two patterns described above was present in $>60 \%$ of snores analysed.

\section{Discussion}

In the present study, the time domain and spectral patterns of snores were explored in sleeping subjects with "heavy" snoring, in a dog model of upper airway obstruction, and in simulated snores generated by awake healthy volunteers. Tracings of snoring sounds and their spectra have been presented in previous studies $[6,7]$ but with little or no analysis of their properties. Recently, a detailed analysis was presented, where the sound data are similar, with emphasis on the location of sound production (nasal $v s$ oronasal) and the characteristics of the first postobstructive snore [8]. The foci of the present study are on sorting out the variety of the sounds, formulating the relationships between the sounds in the time and in the frequency domains, and on analysis of the implications of the temporal correlation between the acoustic properties and airflow on the mechanisms of snoring sound generation.

The time domain pattern of snores changes continuously within a subject during a night's sleep, between and within sleep stages, and even within a breath [8]. However, after observing hundreds of snores we were able to identify two dominant patterns, which are distinctly different from each other: the simple-waveform and the complex-waveform. Both patterns were usually found in every subject. Occasionally, one type gradually or abruptly changed to the other, even within a single breath. Many variants of the two types were observed in the three experimental settings used in this study, and at times it became difficult to classify the sounds correctly. In addition, the snoring sound structures were often embedded in other breathing noises and were difficult to extract from the time tracings. However, the distinct spectral pattern of snores was found useful in identifying a particular sound as snore. Also of interest is the finding that these characteristics of the snoring sounds were preserved irrespective of the microphone's location, ambient, trachea or chest (chest data not included in this study).

\section{Complex-waveform snores}

Time and spectral tracings of the complex-waveform snores are shown in figures $3 \mathrm{a}, 4$ and 5a. They are characterized as a repetitive, equally-spaced, train of sound structures, each composed of a few oscillations, starting with a large deflection which is followed by a decayingamplitude wave. The analysis of the simulated snores tracings, where flow was also recorded, showed that the onset of the major waveform in each sound structure coincided with the peak of the flow signal. The implications of this temporal relationship between the sounds and the flow on the mechanism of snore sound generation are discussed below.

The power spectrum of the complex-waveform snores may be characterized as a sequence of equally-spaced sharp and narrow peaks of power. The amplitude of the peaks has a maximu, with lower amplitude peaks on both of its sides (see figs. 4 panel $4 \mathrm{~b}(\mathrm{M})$ and 5a lower panel). The frequency interval between the peaks was found to be equal to the rate of appearance of sound structures in the snores. The position of the highest amplitude peak was found to represent the frequency of the oscillations within the sound structure. In those cases where there was more 
than one dominant frequency within the structures, there was a corresponding number of maximal amplitude peaks (e.g. fig. 4 panel $2 \mathrm{~b}$ ). The "complex-waveform" snores, though often of a frequency lower than $75 \mathrm{~Hz}$, were not cut off by the highpass filter used in this study, since they are comprised of a number of higher frequency secondary waves. The filter does not recognize a "cluster" of waves as a single event, reacting only to individual waves.

Figure 6 shows three examples of simulated time tracings and spectra of waveforms generated by a simple mathematical model. Figure 6a is a train of waveforms generated from the formula:

$$
\mathrm{Y}=\sin \left(2 \pi f t^{\prime}\right) \cdot \mathrm{e}^{(t / \tau)}
$$

where $f$ is the frequency of the oscillation within the structure, $t$ is the time and $\tau$ is the time constant of the exponential decay of the intensity. The spectrum of this tracing is shown in the lower panel, where the frequency of the highest peak corresponds to $f$ and the spacing between the peaks to the rate of structures within the tracing.

The tracing in figure $6 \mathrm{~b}$ was generated by a slightly more complex formula:

$$
\mathrm{Y}=\sin \left(2 \pi f t^{\prime}\right) \cdot\left[\mathrm{e}^{(t / \tau 1)}+\mathrm{e}^{(t / \tau 2)}\right]
$$

where $\tau 1 \gg>2$. This modification results in a relatively more intense first deflection in the time domain tracing, as seen in many of the experimental data, and wider peaks in the spectrum (figure $6 \mathrm{~b}$, lower panel).

Figure $6 \mathrm{c}$ shows a tracing generated by using a combination of two sine waves, each bounded by its own exponent:

$$
\mathrm{Y}=\sin \left(2 \pi f 1 t^{\prime}\right) \cdot \mathrm{e}^{(t / \tau 1)}+\sin \left(2 \pi f 2 t^{\prime}\right) \cdot \mathrm{e}^{(t / \tau 2)}
$$

The spectrum of the latter waveform (fig. 6c, lower panel) shows two maxima of peak amplitudes, each centred around frequency values corresponding to $f 1$ and f2. Waveforms and spectra generated from formulaes 1-3 correlate closely with the waves and spectra of complexwaveform snores. The implications are discussed below.

\section{Simple-waveform snores}

In the time domain, simple-waveform snores have a quasi-sinusoidal pattern and almost no secondary internal oscillations within the periodic wave. The range of appearances of the simple waveform snores includes nearly true sine wave (fig. 5c), asymmetric periodic wave (fig. 5b), and periodic wave with a secondary deflection (fig. 3b). The frequency of the simple-waveform oscillations was $174-292 \mathrm{~Hz}$, a higher value than the $64-135 \mathrm{~Hz}$ rate of the sound structures in the complex-waveform snores. The spectra of the simple-waveform snores had only 1-3 peaks of power, of which the first one was always the most prominent. However, there were only few tracings with clear sinusoidal waves so that spectra which had only a single peak were rare. This is a clear distinction from the musical sounds of stridor and wheezes, which are usually sinusoidal and represented by a single spike in the spectrum [9]. The distinction between simple-waveform snores and wheezes, which may also have more than one peak of power, is based on the spacing between the peaks. Whilst in the spectra of simple-waveform snores the peaks are equally spaced, the peaks of wheezes are usually not.

\section{The mechanisms of snoring sound generation}

In a recent study, GAVRIELY and JeNSEN [10] developed a simplified theoretical evaluation of the flow-induced

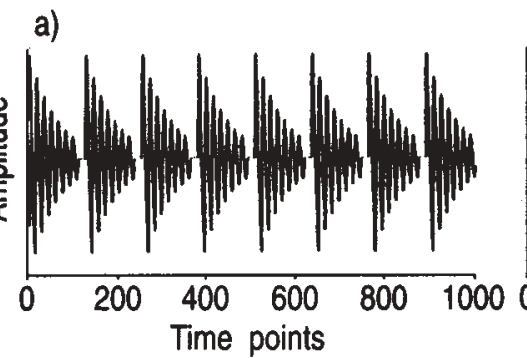

$$
\text { b) }
$$

c)
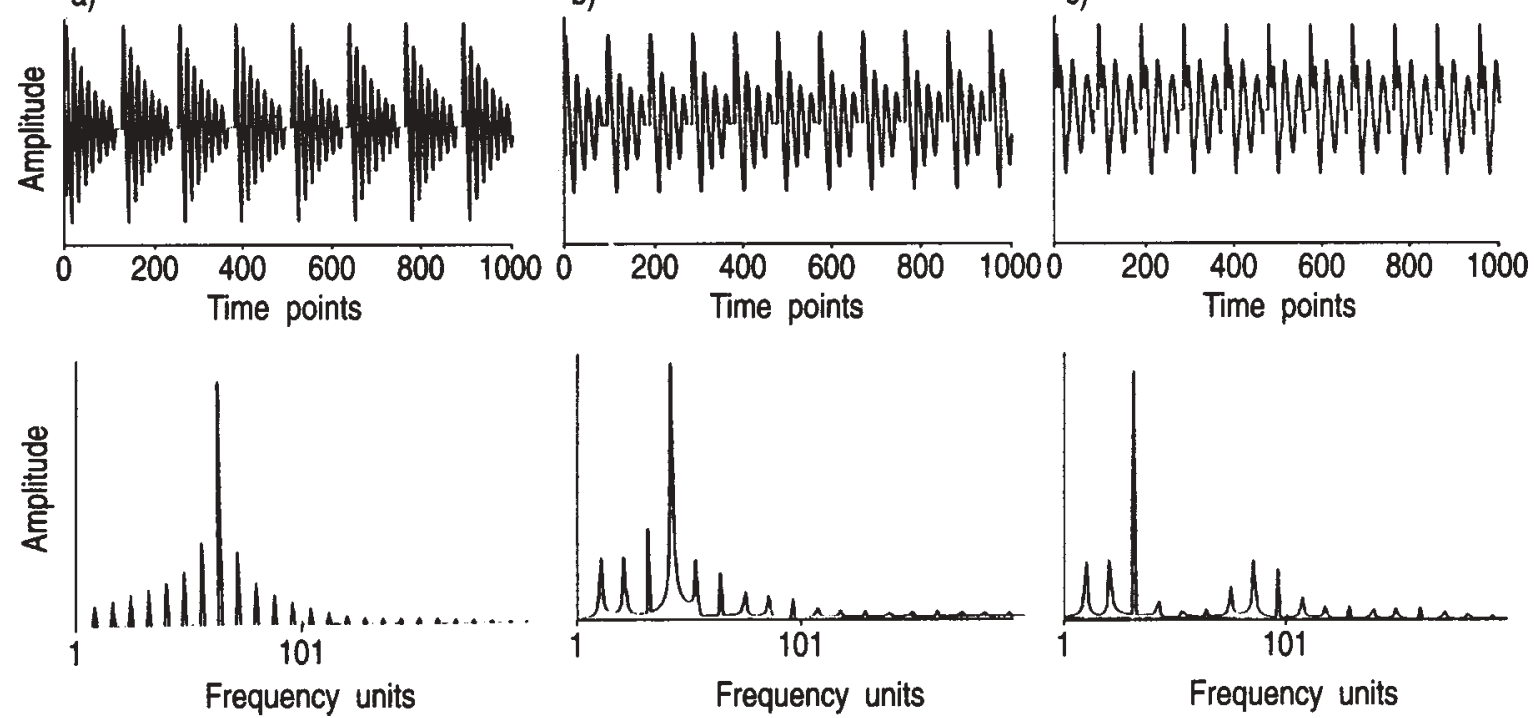

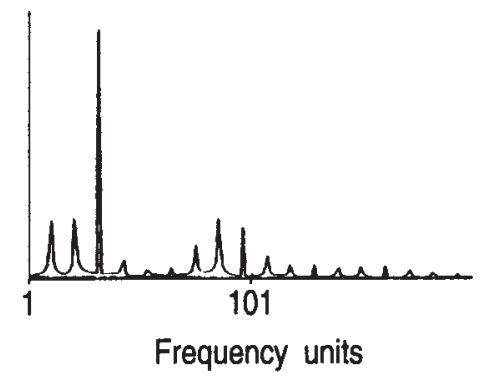

Fig. 6. - Mathematical representation of snoring waveforms. The upper panels show the waveform resulting from the formula and lower panels the frequency analysis (fast fourier transform (FFT)) of that waveform. a-c) progression from "simple- to "complex"-waveforms and their spectra (see text for details). Note the matching of these waveforms and their spectra to those obtained from the dog model (fig. 3), simulated snores (fig. 4) and snoring sleeping humans (fig. 5). 
airway wall motion in the upper airways. The mathematical model predicted that under certain circumstances, determined by the dimensions of the airway (diameter, width and length of the movable section), the flow rate, the upper airway resistance, the airway wall compliance and the density of the gas, the airway walls move rapidly towards each other and the airway will close in a slam. Under other circumstances, (i.e. other values of the above listed parameters), the movable section of the wall will oscillate around a neutral position without reaching actual closure. In the first case, the two opposing walls collide and the airway lumen becomes occluded for a brief period of time. The forces and mechanisms of the reopening of the airway have not yet been analysed, but if such reopening is induced and subsequently followed by another closure event, a cycle of closure-reopening events is expected. From the analysis of the dynamics of the closing event it is predicted that the colliding wall will move in an accelerating rate and is likely to release acoustic energy. Our present data and the closely matching mathematical model correlate well with this theory: the complex-waveform snores represent, or are the result of, the slamming closed of the airway walls, whereas the simplewaveform snores represents vibration without closure of the airway.

In analysing the time relationship of the flow and snoring sound signals in our study, some methodological issues need to be considered. The frequency response of the Validyne transducer is slow compared to the microphone and contact sensor. In our data, analysis of the temporal relationships between the sound structures and the flow signal in the simulated snores indicates that the onset of the major component of the sound structure always coincides with the maxima of the flow signal. However, because of the different frequency responses of the devices, we can only conclude that there is a close and constant relationship between the sound and flow events. Thus, there are two possible mechanisms for production of the snoring sound: 1) slamming shut of airway walls with resulting tissue vibration; 2) and rapid popping open of the shut lumen with aeroelastic interactions, similar to those observed in speech [8]. However, unlike speech, where the sound is generated by motion of the vocal cords, the location of the moving structure is supraglottic [11]. Both mechanisms invoke closure of the lumen as a requirement, however, in none of the tracings has there been a recording of zero flow. This relates back to the characteristics of the Validyne transducer. We found that the recorded flow value at the minima was dependent on the rate of generation of sound structures. In the slowest rate tracings, the flow was close to zero (fig. 4 panel 2). It was further away from zero in the tracings that showed higher rates of sound structures (fig. 4 panel 1). In none of the flow tracings did we actually observe zero flow, as expected if the airway is completely occluded. However, the frequency response of the differential pressure transducer used with the pneumotachograph in our study, which is around $35 \mathrm{~Hz}$ [12], and the cut-off frequency of the lowpass filter induced by the compliant upper airways, which is even lower than $35 \mathrm{~Hz}$ [13], make it impossible for the system to show a recording of zero flow, if the flow oscillation rate is greater than about $30 \mathrm{~Hz}$. Further studies will be needed to clarify this point.

It should also be noted that wall oscillations with closure of the lumen is a distinctly different mechanism from the "flutter" mechanism described for wheezes [14, 15], and is not necessarily associated with true airway flow limitation ("airway flow limitation" in its strict physical meaning, namely, that increasing driving pressure does not cause any increase in flow). The repetitive closure and reopening of the airway may well impede the flow but the flow rate may still be dependent on the driving pressure. This conclusion is in contrast to the theory presented by Perez-PADILla et al. [8], who concluded that snoring sound generation fits best with the "flutter" theory. Our findings support their alternative theory of "relaxation oscillations" [16, 17].

This analysis of our data leads to the conclusion that complex-waveform snores are associated with upper airway wall oscillations with actual intermittent closure of the lumen flapping flutter). The simple-waveform snores are higher frequency sounds that are probably associated with aeroelastic interactions between the airflow and the airway wall without actual closure of the lumen $[14,15]$. Thus, whilst both wheezes and snores are aeroelastic phenomena, where airway wall motion plays a dominant role in sound production, their differences have to do with the amplitude of wall oscillation and its mechanical properties: in wheezes the wall motion amplitude is small relative to the lumen diameter; whereas, in snores the amplitude of wall motion is large and may reach complete momentary closure in complexwaveform snores. Simple-waveform snores are probably only associated with partial closure.

\section{References}

1. Lugaresi E, Cirignotta F, Coccagna G, Piana C. Some epidemiological data on snoring and cardiocirculatory disturbances. Sleep 1980; 3: 221-224.

2. Lugaresi E, Coccagna G, Mantovani M. Hypersomnia and periodic apneas. In: Advances in Sleep Research. Jamaica, NY, Spectrum Publications, 1978; 4: pp. 1-151.

3. Cohen A, Bernstein AD. Acoustic transmission of the respiratory system using speech simulation. IEEE Trans Biomed Eng 1991; 38(2): 126-132.

4. Lieberman A, Cohen A. Computerized analysis of snoring. In: Chouard CH, ed. Chronic Rhonchopathy. London/Paris, John Libbey Eurotext Ltd, 1988; pp. 73-77.

5. Block AJ, Faulkner JA, Hughes RL, Remmers JE, Thach B. Factors influencing upper airway closure. Chest 1984; 86: 114-122.

6. Liistro G, Stanescu D, Veriter C. Pattern of simulated snoring is different through mouth and nose. J Appl Physiol 1991; 70: 2736-2741.

7. Perez-Padilla JR, Remmers JE. Dynamics of pressure, airflow and noise production during simulated snoring. Am Rev Respir Dis 1986; 131: A106 (Abstract).

8. Perez-Padilla JR, Slawinski E, Difrancesco LM, Feige RR, Remmers JE, Whitelaw WA. Characteristics of the snoring noise in patients with and without obstructive sleep apnea. Am Rev Respir Dis 1993; 147: 635-644. 
9. Gavriely N, Palti Y, Alroy G, Grotberg JB. Measurement and theory of wheezing breath sounds. J Appl Physiol 1984; 57(2): 481-492.

10. Gavriely N, Jensen O. Theory and measurement of snores. J Appl Physiol 1993; 74: 2828-2837.

11. Remmers JE, Feroah T, Perez-Padilla JR, Whitelaw WA. Correlation of structure and mechanics in pharyngeal obstmction dunng sleep. In: Chouard $\mathrm{CH}$, ed. Chronic Rhonchopathy. London/Paris, John Libbey Eurotext Ltd, 1988; pp. 30-35.

12. Francis G, Gelfand R, Peterson RE. Effect of gas density on the frequency response of gas-filled pressure transducers. J Appl Physiol: Respirat Environ Exercise Physiol 1979; 47(3): 631-637.
13. Fredberg JJ, Hoenig S. Mechanical response of the lung at high frequencies. J Biomech Eng 1978; 100: 57-66.

14. Gavriely N, Shee TR, Cugell DW, Grotberg JB. Flutter in flow-limited collapsible tubes: a mechanism for generation of wheezes. J Appl Physiol 1989; 66(5): 2251-2261.

15. Grotberg JB, Gavriely N. Flutter in collapsible tubes: a theoretical model of wheezes. J Appl Physiol 1989; 66(5): 2262-2273.

16. Bertram CD. Two modes of instability in a thick-walled collapsible tube conveying a flow. J Biomech 1982; 15: 223-224.

17. Cancelli C, Pedley TJ. A seperated flow model for collapsible tube oscillations. J Fluid Mech 1985; 157: 375-404. 